\title{
The North American Society of cardiovascular imaging
}

\author{
Jacobo Kirsch $\cdot$ Amgad N. Makaryus
}

Received: 27 May 2013/Accepted: 30 May 2013/Published online: 8 June 2013

(C) Springer Science+Business Media Dordrecht 2013

We at the North American Society for Cardiovascular Imaging (NASCI) are very proud of our long and fruitful relationship with the International Journal of Cardiovascular Imaging. As the official journal of the society, the publication has promoted cardiac and vascular multimodality imaging across our ranks.

NASCI was established in 1972 with the specific goal of advancing the science and art of cardiac radiology. Forty years later, the mission of the Society has been expanded to:

- Encourage the development and dissemination of knowledge regarding Cardiovascular Imaging.

- Contribute to the active continuing education of its membership and the Medical Community.

- Promote the study, research and clinical practice of Cardiovascular Imaging.

Through its on-line offerings, NASCI is proud to further push its goals to the greater imaging community by providing a significant portion of its content for free, such as bi-weekly interesting case presentations and a real-time journal-watch with updates on the most interesting publications that relate to the specialty.

For the last 4 years, The Prepared Minds section of the website has been selecting what its Editorial Board suggests as must-read articles in cardiovascular imaging. The Board is made up of imagers from backgrounds in both Cardiology and Radiology from several countries, which allows for a very broad source of references to recommend. As publications are recommended, the Editors submit comments about the paper, and readers (as well as the paper authors) are always invited to participate in that conversation.

One of the most exciting features of the section is the monthly selection of a journal club paper. At the beginning of every month a paper is singled out as ideal for discussion in a journal club setting. Additionally, a guideline for reviewers on how to organize a journal club and questions/ topics specific to the selected paper are provided. Institutions and groups that take advantage of this tool are also invited to post their journal club conclusions on the site for other readers to read.

Readers of the International Journal of Cardiovascular Imaging are familiar with the published Case-in-Point section, started 5 years ago. These interesting cases are selected from the pool of interactive case presentations on the NASCI website. Every 2 weeks, a new case is posted on-line featuring still and video images as well as educational facts. We have recently upgraded our submission format to make it easier to prepare the cases and to allow for a larger number of them to translate to peer-review publications in this journal.

We invite the entire readership to visit the NASCI website and take advantage of all the educational material available. There has never been a better time to become a member and participate in the NASCI-sponsored activities.

J. Kirsch $(\square)$

Cleveland Clinic Florida, Weston, FL, USA

e-mail: kirschj@ccf.org

\section{A. N. Makaryus}

North Shore University Hospital, Manhasset, New York, USA 